\title{
HoxA Genes and the Fin-to-Limb Transition in Vertebrates
}

\author{
João Leite-Castro ${ }^{1}$, Vanessa Beviano ${ }^{1,2}$, Pedro Nuno Rodrigues ${ }^{1,2,3}$ and Renata Freitas ${ }^{1,2, *}$ \\ 1 IBMC-Instituto de Biologia Celular e Molecular, Oporto, Portugal; newtonjplc@hotmail.com (J.L.-C.); \\ vanessa.beviano@hotmail.com (V.B.); PRodrigu@ibmc.up.pt (P.N.R.) \\ 2 I3S-Instituto de Investigação e Inovação em Saúde, Rua Alfredo Allen, 208, 4200-135 Porto, Portugal \\ 3 ICBAS-Instituto de Ciências Biomédicas Abel Salazar, University of Oporto, Rua de Jorge Viterbo \\ Ferreira $n^{\circ}$. 228, 4050-313 Oporto, Portugal \\ * Correspondence: renata.freitas@ibmc.up.pt; Tel.: +351-220-408-800
}

Academic Editors: Vincenzo Zappavigna and Andy Wessels

Received: 1 December 2015; Accepted: 4 February 2016; Published: 17 February 2016

\begin{abstract}
HoxA genes encode for important DNA-binding transcription factors that act during limb development, regulating primarily gene expression and, consequently, morphogenesis and skeletal differentiation. Within these genes, HoxA11 and HoxA13 were proposed to have played an essential role in the enigmatic evolutionary transition from fish fins to tetrapod limbs. Indeed, comparative gene expression analyses led to the suggestion that changes in their regulation might have been essential for the diversification of vertebrates' appendages. In this review, we highlight three potential modifications in the regulation and function of these genes that may have boosted appendage evolution: (1) the expansion of polyalanine repeats in the HoxA11 and HoxA13 proteins; (2) the origin of $+a$ novel long-non-coding RNA with a possible inhibitory function on HoxA11; and (3) the acquisition of cis-regulatory elements modulating 5' HoxA transcription. We discuss the relevance of these mechanisms for appendage diversification reviewing the current state of the art and performing additional comparative analyses to characterize, in a phylogenetic framework, HoxA11 and HoxA13 expression, alanine composition within the encoded proteins, long-non-coding RNAs and cis-regulatory elements.
\end{abstract}

Keywords: HoxA genes; transcriptional regulation; development; evolution; limb; fin

\section{Introduction}

The autopod, the multi-fingered extremity located at the end of a hindlimb or forelimb, is a specific characteristic of tetrapods [1-3]. The fossil record suggests that this anatomical structure evolved from sarcopterygian fins by sequential expansion and elaboration of their endochondral bones and concomitant reduction of the apical dermoskeleton [1-3]. Regarding the number of digits, both paleontological and developmental data suggest an evolutionary sequence that went from an adactyl state detectable up to the divergence of sarcopterygians [4], then sidestepping by digit-like radials in stem sarcopterygians [5], polydactyly in stem tetrapod and culminating in the fixation of pentadactyl [6-8]. From a developmental point of view, understanding the molecular mechanisms that enabled the fin-to-limb transition is a puzzling question that has long held the attention of the scientific community and holds the potential of enlightening molecular biologists on how gene regulatory networks and interactions evolved.

The patterning of the skeletal elements, in both fin and limb, largely relies on the action of signaling centers operating from the onset of their outgrowth: the apical ectodermal ridge (AER) and the zone of polarizing activity (ZPA) [9]. However, there are marked differences in the morphology 
and development of the most distal part of fins and limbs. In tetrapods, the influence of the AER is maintained throughout limb development, being important for the differentiation of the stylopod, zeugopod and autopod [10-12]. In fish, however, this structure is quickly converted into a finfold (FF) during fin development that then gives rise to the apical dermoskeleton [13]. However, its function seems to be conserved during fish fin development and relates to the coordination of the proximo-distal patterning [14]. Recent studies performed in zebrafish suggest that the timing of the AER-FF transition may have mediated the differences found between fins and limbs and that the acquisition of a mechanism repressing the formation of the FF may have been crucial in the development of tetrapod limbs [15].

The patterning of the endoskeleton structure has been proven to rely on the expression of $5^{\prime}$ HoxA and HoxD genes in both fins and limbs, as demonstrated by different studies performed on a wide variety of model systems [16-20]. Indeed, several lines of evidence highlight the role of these transcriptional factors as key intervenients in limb development. For example, loss-of-function mutations in genes located at the $5^{\prime}$ end of the mouse HoxA and HoxD clusters lead to the appearance of limbs with atavistic characters $[8,18,20]$. The impact that these genes have on the patterning of the limb, proven by loss-of-function assays, led Duboule and colleagues to suggest that modifications on Hox regulation may have been a source of morphological variation during the evolution of tetrapod limbs [21]. Moreover, they suggest that increased levels of these transcription factors during fin development may have caused extra-proliferation distally, setting the grounds for the formation of additional endoskeleton elements, which occurred concomitantly with the reduction of the apical dermoskeleton. To make the proof of principle of these ideas, Freitas and colleagues overexpressed the zebrafish counterpart of HoxD13 (hoxd13a), generating a fin phenotype characterized by additional endochondral tissue and reduction or ablation of the apical finfold [22]. Thus, hoxd13a overexpression induces a phenotype that mirrors two of the major morphological alterations marked as crucial in limb evolution by the fossil record: endochondral expansion and dermoskeleton retraction [1,22].

Taking into account the results obtained by Zákány et al. [8] and Freitas et al. [22], it seems that both HoxA and HoxD clusters contributed to the fin-to-limb transition, although at distinct time points and by regulating different cellular events. Zákány and colleagues analyzed the phenotypes of several compound mouse mutants with distal HoxA and HoxD loss-of-function alleles and proposed that the HoxA13 gene was the initial/primordial major contributor for the determination of the autopod identity [8]. This was probably followed, in the course of evolution, by the recruitment of distal HoxD genes (HoxD11, HoxD12 and HoxD13), whose expression is associated with finger elongation and fixation of $[8,23]$. Further evidences that supports this sequence of events comes from the observation that HoxA13 is expressed earlier than HoxD13 in the distal mesenchyme during limb development [23], being probably an earlier specifier of the autopod identity. Moreover, the expression of the Hox 13 paralogs in zebrafish is detected in the most distal mesenchyme of the developing fins, closely mirroring the expression patterns described in tetrapods [17]. This suggests that HoxA13 was already essential to induce a distal appendage identity even prior to the origin of the autopod in the tetrapod lineage. Taken together, these data suggest that alterations in the expression and function of HoxA13 may have been the primary mechanisms leading to the formation of the autopod in the tetrapod lineage $[24,25]$.

Taking into account the conserved hoxa13 expression patterns found distally in developing fish fins, why do they not give rise to autopod-like structures? Information gathered from developmental, phylogenetic and gene function studies suggests that, in the course of evolution, two factors had to meet to allow the formation of an autopod domain, those being the alteration of the proximo-distal patterning leading to the formation of novel distal territories, characterized by a specific transcriptome, that co-occurred with, or was followed by, an increase in the levels of HoxA13 transcripts [8,26].

Regarding the proximo-distal patterning, striking differences have been found in the expression of $5^{\prime}$ HoxA genes in fish and tetrapod models that may underlie an increased endoskeletal complexity throughout fin/limb evolution (Figure 1). This process counts with the combined action of three genes encoding master regulators of transcription: Meis1, which seems to be essential for stylopod specification; HoxA11 required for proper zeugopod development; and HoxA13, which contributes 
to an autopod identity [27-31]. Interestingly, during tetrapod limb development, the initially overlapping expression domains of HoxA11 and HoxA13 split up to a point in which HoxA11 is exclusively expressed in the prospective zeugopod, while HoxA13 is restricted to the future autopod (Figure 1) [16,32,33]. The expression patterns of these genes during fish fin development are, however, quite different. In zebrafish, a full separation of the hoxa11 and hoxa13 expression domains has never been reported in the developing fins $[17,21,34]$. In other fish representing distinct phylogenetic positions, such as chondrichthyans (catshark; [35]) and basal actinopterygians (paddlefish; [26,36]), there is only a transient separation of hoxa11 and hoxa13 expression domains during early stages of fin development. These data suggest that the decoupling of HoxA13/HoxA11 expression domains may have occurred in the common ancestor of gnathostomes and was secondarily lost in a particular lineage of actinopterygians, the teleosts.

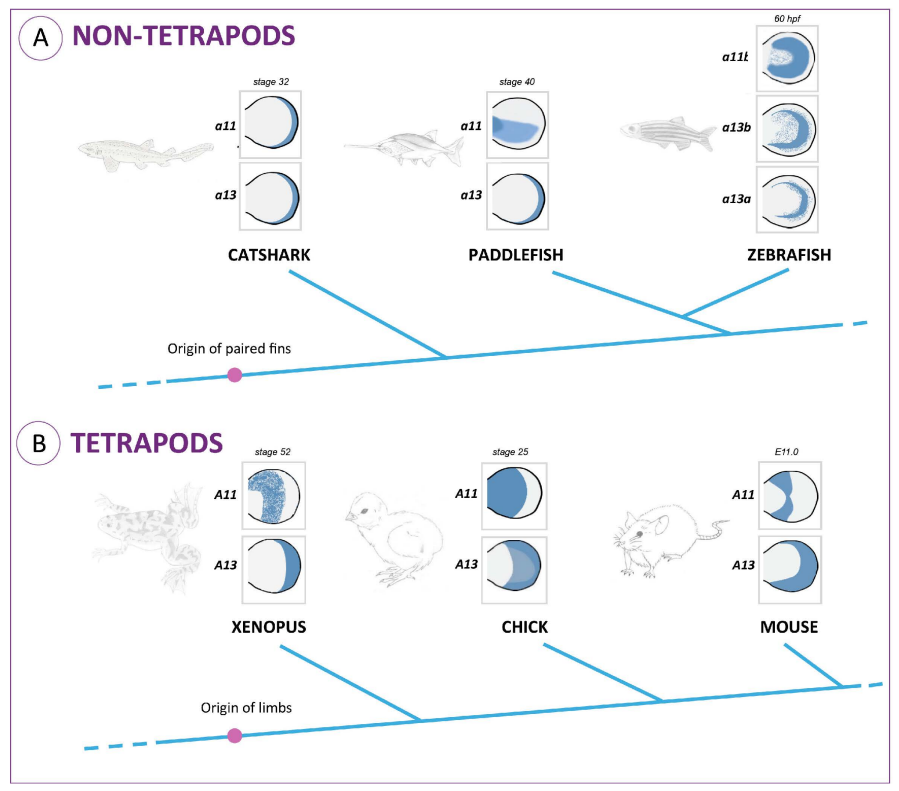

Figure 1. Expression patterns of $5^{\prime}$ Hox $A$ genes during the development of paired appendages in vertebrates. The selection of stages took into account data from: Xenopus [16]; chick [23]; mouse [31]; catshark [35]; paddlefish [36]; zebrafish [17]. (A) Expression domains of HoxA11 and HoxA13 in developing fins from fish representing phylogenetic groups that diverged prior to the origin of tetrapods; (B) Expression domains of HoxA11 and HoxA13 in developing limbs in representatives of tetrapod lineages (in blue).

The origin of a distal domain exclusively expressing HoxA13 may have influenced the formation of novel endoskeleton structures [26,37,38]. Further support of this hypothesis is provided by regeneration studies in Xenopus, where non-separation of HoxA11 and HoxA13 expression patterns, making distal blastemal cells HoxA11 and HoxA13 double positive, appeared to be associated with the inability to regenerate an autopod [16]. Interestingly, HoxA11 and HoxA13 mRNA are co-expressed in the developing limbs of axolotls, but the translated HoxA11 and HoxA13 proteins are distributed in distinct domains consistent with the formation of a zeugopod-autopod border [39]. Questions that remain to be answered are, for example: How was the separation of HoxA11 and HoxA13 domains achieved, and what were the implications for the evolution of the limb morphology? What were the molecular mechanisms associated with this alterations? Or, how did alterations in HoxA11 and HoxA13 protein levels contribute to the autopod origin?

From a gene regulatory perspective, there are several mechanisms that could have contributed to the establishment of a HoxA13 single positive distal appendage domain that allowed the formation and posterior evolution of the autopod. These may include: (1) alteration in the signaling 
pathways that act upstream of HoxA13, regulating its expression and/or the expression of HoxA11; (2) remodeling/alteration or elaboration of the cis-regulatory motifs that assume the spatial-temporal control of the expression for these genes; (3) acquisition/loss of elements of the post-transcriptional gene regulatory network; (4) alteration of the protein-protein or protein-DNA interactions through alteration of the coding sequence.

By analyzing the reports published to date, there are three mechanisms that may uphold an evolutionary relevance in the context of the fin-to-limb transition, those being the acquisition/expansion of HoxA11 and HoxA13 homopolymeric repeats, acquisition of a new long-non-coding RNA with possible HoxA11 inhibitory function and the origin of novel cis-regulatory regions acting on HoxA11 and HoxA13 [40-43]. Here, we will explore the evolutionary/developmental relevance of these three mechanisms, reviewing the current state of the art concerning HoxA11 and HoxA13 regulation throughout development in species at different phylogenetic positions and discussing how these developmental regulatory networks may have evolved, being at the core of the morphological diversification found in vertebrate appendages.

\section{Polyalanine Repeats and the Fin-to-Limb Transition}

Amino acid repeats are common among eukaryote proteins, and their length and frequency cannot be explained solely by random chance, suggesting a biological function and positive selection $[44,45]$. Interestingly, prokaryote proteins are impoverished in amino acid repeats, when compared to their eukaryotic homologues, indicating that evolution of such amino acid repeats happened after establishment of the initial protein structures and possibly concomitantly with the appearance of novel functionalities in eukaryotes [44]. Indeed, numerous proteins with a variety of different biological functions display amino acid repeats, such as protein kinases, signaling proteins, membrane transporters and transcription factors [44]. Strikingly, the majority of amino acid repeats detected in eukaryotes are present in proteins involved in the formation of large protein-protein or protein-nucleic acid complexes, such as transcriptional factors, possibly influencing their performance [44]. Thus, changes in these particular sequences during evolution may have produced a significant impact, leading to the genesis of novel morphological structures, for example.

Interestingly, alterations in the length of amino acid tandem repeats have long been suggested as a major source of phenotypic variation in evolution [46]. This hypothesis was constructed based on the observation that: (1) amino acid tandem repeats are abundant and frequently conserved in the coding sequence of genes involved in development; (2) repeat length variation, both expansion and contraction, occurs in a locus-specific manner 100,000-times more frequently than point mutations; and (3) transcription, mRNA processing, protein synthesis and morphology are affected by length variation in the amino acid repeats [46]. There are, indeed, some examples that offer support to this hypothesis. For example, within dog breeds, there is a clear association between the contraction of glutamine repeats in the $A l x-4$ gene and the development of bilateral polydactyly that characterizes the Great Pyrenees breed [46]. Moreover, a correlation was found between the length of the polyglutamine and polyalanine tracts in the Runx-2 gene and the facial morphology of different dog breeds [46], showing the potential that amino acid repeat variability may assume in the diversification of phenotypes.

Polyalanine repeats are types of amino acid repeats characterized by tandem repeated alanine residues [44]. These regions can modulate protein function through various mechanisms, such as enabling or increasing a transcriptional factor capacity to act as a repressor, modifying the interactome of a protein or acting as spacers, merely providing a structural component that enhances the performance of its functional domains [40,47-49]. Interestingly, alterations in the alanine tracts of particular Hox proteins have been proven to impact limb development, to a greater or lesser extent [50,51]. For instance, deletion of one of the HoxD13 alanine polymers in mouse caused fusion of the forelimb sesamoid bones $[51,52]$. Moreover, increased length of HOXD13 polyalanine repeats has been associated with several cases of synpolydactylism [51,53]. 
HoxA11 and HoxA13 are among the transcriptional factors with crucial function during limb development, where polyalanine repeats have been identified [40,41]. For the human HOXA13, in particular, clear associations were established between the expansion of particular polyalanine tracts (I, III and V) and the development of autopod malformations associated with the human hand-foot-genital syndrome (HFGS) [54,55]. Similar phenotypes were identified in mutant mice with an expanded polyalanine tract [56].

Comparative analyses using HoxA11 amino acid sequences from vertebrates reveal that this protein is composed of three major domains [41]: Domains I and III correspond to the N- and C-terminal regions respectively; Domain II is encoded by exon 1 and corresponds to a variable and overall hydrophilic region (Figure 2A). Characteristic reconstructions of Domains I and III suggest that the rates of coding sequence evolution have not changed significantly in tetrapods (frog and chick) relative to lobe-finned fish (coelacanth). However accelerated rates of coding sequence evolution were observed for the mammalian and newt lineages and shown to be a gene-specific phenomenon [41]. Interestingly, when the hydrophilic region of the HoxA11 proteins is compared, stretches of more than three consecutive alanine residues are identified exclusively in the tetrapod sequences, together with an increase in the length of this region (Figure 2B). Thus, the molecular evolution of this transcription factor might have involved an increase of the hydrophilic region by the addition of polyalanine tracts. An hypothetical scenario is that the alanine repeats present in the common ancestor of tetrapods may have acted as a "seed" for the expansion of polyalanine stretches that then contributed to an increased plasticity of HoxA11, potentiating novel functionalities in this lineage, ultimately contributing to the developmental events involved in the fin-to-limb transition [41].

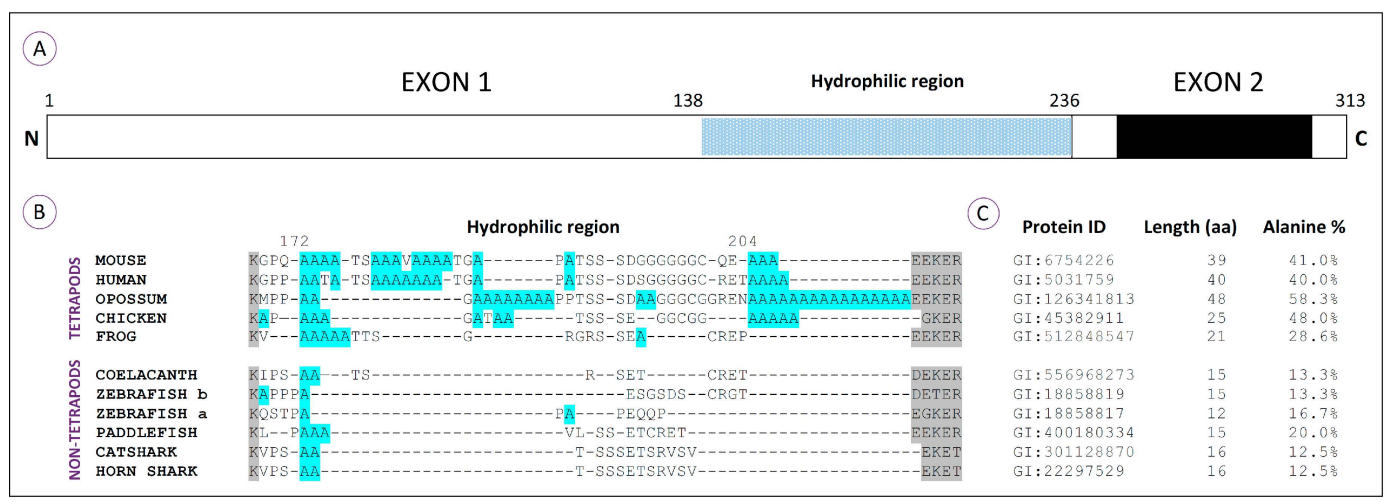

Figure 2. Structure and alanine content of the HoxA11 proteins from vertebrates. (A) Schematic representation of the HoxA11 protein, which has 313 aa (amino acid) based on mouse [41,57]. Exons 1 and 2 are represented by white rectangles; the homeobox is in black; and $\mathrm{N}$ and $\mathrm{C}$ terminal ends are indicated. The variable overall hydrophilic region (98 aa) identified in the N-terminal region is represented by a dotted bar; (B) Amino acid sequence alignment of the presumptive hydrophilic region of HoxA11 proteins in vertebrates: mouse (Mus musculus), human (Homo sapiens), opossum (Monodelphis domestica), chicken (Gallus gallus), frog (Xenopus tropicalis), coelacanth (Latimeria chalumnae), zebrafish (Danio rerio), paddlefish (Polyodon spathula), catshark (Scyliorhinus canicula) and horn shark (Heterodontus francisci). Alignments were performed with Clustal X [58]. Conserved amino acid residues, flanking the region, are shadowed in grey and alanine residues in blue. Note the presence of polyalanine tracts, with more than four alanine residues clustered, exclusively in tetrapod organisms; (C) Identification of the HoxA11 proteins used in the alignment (Protein ID) and the corresponding alanine percentage within the presumptive hydrophilic regions. The sequences used to calculate the alanine percentage correspond to blocks located between extremely conserved residues (in grey). The length of the compared blocks is also indicated here (aa). Note a significant increase of these blocks and a higher percentage of alanine residues in tetrapod organisms, particularly evident in amniotes. Within non-tetrapods, the higher percentage of alanine is found in paddlefish. 
Moreover, the overall alanine content within the hydrophilic region is higher in tetrapods than in non-tetrapod organisms. This is especially evident in amniotes, animals that develop a paddle-like hand plate prior to digit differentiation. In fact, the amphibian's hydrophilic region lacks polyalanine tracts that appear to be conserved in other tetrapod lineages, and coincidently, the formation of their autopods is not preceded by the development of a hand plate $[59,60]$. Thus, the addition of alanine residues in the hydrophilic region may have led to novel functionalities of the HoxA11 protein that may have contributed to the origin of the hand plate. Alternatively, the lower alanine content found in the hydrophilic region of amphibians, when compared to other tetrapod linages, may reflect their own evolutionary history that seems to have led to a highly derived morphology [61].

Interestingly, in non-tetrapod organisms, the higher percentage of alanine residues in the hydrophilic region is found in the paddlefish, which in fact presents a subtle separation of HoxA11 and HoxA13 expression domains at early stages of fin development (Figure 1A; [36]). Thus, hypothetically, higher alanine content in the HoxA11 hydrophilic regions may influence the expression pattern of this protein. Evolutionarily, this could have been the process by which HoxA11 expression patterns evolved, probably contributing to the fin-to-limb transition.

Given that the repressor domains of transcription factors are commonly small, unstructured, hydrophilic regions with high alanine content [62], these observations suggest that expanded alanine stretches might be enhancers of repressor domains that were instrumental in setting tetrapod-specific features, such as the autopods, as proposed by Chiu and colleagues [41]. However, the search of such a repressor function found no evidence in mouse [63]. Nevertheless, there is still grounds for Chiu and colleagues' hypotheses, given that the studies performed in mouse just revealed results for a partial deletion of the alanine residues outside the homeodomain, and there is no indication of their location. Thus, just a complete deletion of the alanine residues within the hydrophilic region would be informative. Moreover, the alanine stretches have other predictable roles, such as increasing the plasticity of HoxA11 function, modifying its interactome and/or acting as spacers, providing a structural component crucial to the performance of its functional domains, as suggested by several authors with respect to the function of polyalanine tracts [40,47-49].

Taking together these analyses, we hypothesize that a sequential increase of the alanine content within the HoxA11 hydrophilic regions may have contributed to: (1) the evolution of HoxA11 expression patterns during fin development; (2) the establishment of zeugopod/autopod borders; and (3) the formation of the hand plate in the common ancestor of amniotes.

Regarding the HoxA13 proteins, a total of seven polyalanine tracts (I-VII), characterized by a minimum of four clustered alanine residues, has been identified in a variety of vertebrate species at distinct phylogenetic positions (Figure 3A,B; $[40,47]$ ). Comparative amino acid analyses reveal that these sequences are characteristic of amniotes, being particularly conserved in mammals (Figure 3B). In fact, Tract III seems to be the only one conserved between mammalian and avian genomes, which suggests that most polyalanine tracts from the HoxA13 proteins arose after the divergence of birds from the lineage that would culminate in the appearance of mammals [40,47]. The incorporation of polyalanine tracts together with flanking segments rich in proline, serine and glycine seems to have increased the $\mathrm{N}$-terminus of mammals approximately $35 \%$, when compared to the fish counterparts [40]. Indeed, while tetrapod HoxA13 proteins have more than $20 \%$ alanine content, the zebrafish orthologs have less than $10 \%$ (Figure 3C). Within other organisms representing groups that derived prior to the origin of amniotes (frog, coelacanth, zebrafish, paddlefish and sharks), there are alanine residues that align with the polyalanine tracts of amniotes and may represent the primordial elements that gave rise to them. In agreement with this idea, Mortlock and colleagues suggested that the origin of large alanine repeats in mammals might be explained by a dynamic process of recurring replication slippage and point mutation within alanine repeat codons [40]. 


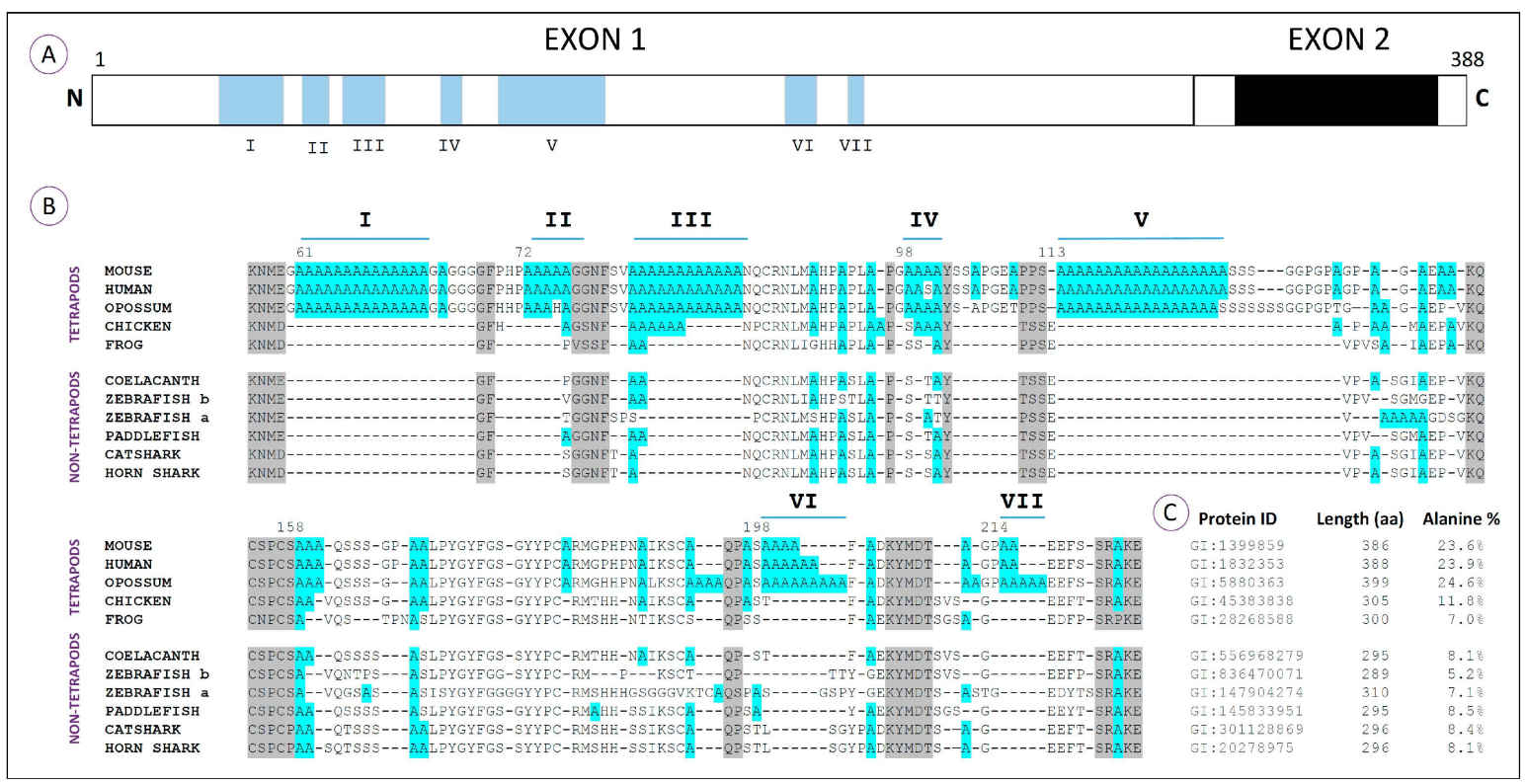

Figure 3. Structure and alanine content of the HoxA13 proteins from vertebrates. (A) Schematic representation of the HoxA13 protein, which has 383 aa (amino acid) based on mouse [47,64]. Exons 1 and 2 are represented by white rectangles; the homeobox is in black; and the $\mathrm{N}$ and $\mathrm{C}$ terminal ends are indicated. Alanine tracts found in the N-terminal region are represented in blue (I-VII); (B) Amino acid sequence alignment showing the alanine residues (in blue) detected in the N-terminal region of HoxA13 proteins in vertebrates: mouse (Mus musculus), human (Homo sapiens), opossum (Monodelphis domestica), chicken (Gallus gallus), frog (Xenopus tropicalis), coelacanth (Latimeria chalumnae), zebrafish (Danio rerio), paddlefish (Polyodon spathula), catshark (Scyliorhinus canicula) and horn shark (Heterodontus francisci). Note the presence of seven polyalanine tracts (I-VII) exclusively in the mammalian Hoxa13 proteins and conservation of Tract III among amniotes; (C) Identification of the HoxA13 proteins used in the alignment (Protein ID) and the corresponding alanine percentage. Note the higher percentage of alanine residues in amniotes, in particular in mammals.

Taking into consideration the potential impact of these polyalanine tracts in the functional plasticity of the proteins, it is possible that their incorporation within the HoxA13 transcription factor may have enabled novel developmental roles contributing to the evolution of the limb structure. However, the absence of such sequences in the HoxA13 of amphibians is intriguing in this scenario. As for HoxA11, we hypothesize that the substantial difference found in the alanine content of the amphibian HoxA13 proteins may explain the lack of a hand plate prior to digit differentiation, which typically characterize the development of amphibian limbs [59]. In order to test this idea, it would be ideal to gain insight into the functional role of Region III, which is conserved in the remaining tetrapod lineages. Several experimental approaches might be relevant to test this hypothesis, including harboring an equivalent region into zebrafish HoxA13, evaluating its capacity to generate a hand plate-like phenotype. Deletions of this particular region in mouse may also be informative to link its functional role to the formation of the hand plate structure.

As for HoxA11, a possible outcome of the modifications in the alanine content of HoxA13 proteins may be the enhancement of their role as repressors. An example of such a type of repressive function is well described for HoxA7, in which $\mathrm{N}$-terminal polyalanine repeats are fundamental to enhance its repressive function [65]. This might have enabled this protein to act as a repressor of the HoxA11 gene during limb development, leading to the establishment of an ancestral zeugopod/autopod boundary and the development of a hand plate. Moreover, dissociation of HoxA11 and HoxA13 expression domains may have potentiated the expansion of the skeletogenic process that culminated in the formation of additional endoskeleton elements, such as digits (Figure 4). 


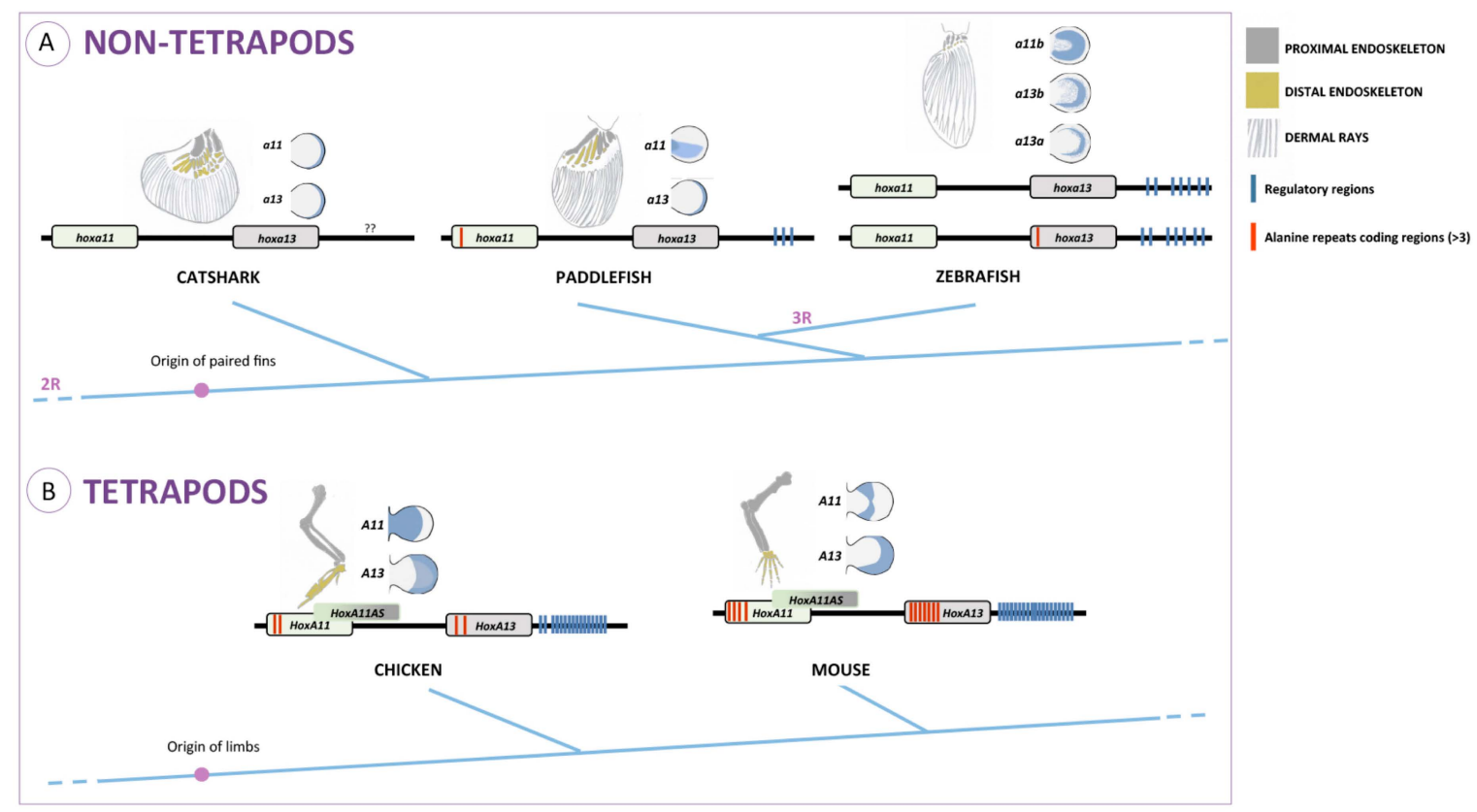

Figure 4. Overview of the morphological, developmental and genomic changes that may have preceded the fin-to-limb transition in vertebrates, comparing non-tetrapod (A) and tetrapod (B) organisms. Schematic drawing of appendicular skeletons from vertebrates representing distinct phylogenetic positions: basal gnathostomes (catshark); basal actinopterygians (paddlefish); teleosts (zebrafish) and tetrapods (mouse and chicken). $2 \mathrm{R}$ and $3 \mathrm{R}$ refer to the number of whole-genome duplications that took place prior to the divergence of gnathostomes and teleosts, respectively. Panels adjacent to the appendicular skeletons display HoxA11 and HoxA13 expression during appendage development (in blue) based on data from: Xenopus [16]; chick [23]; mouse [31]; catshark [35]; paddlefish [36]; zebrafish [17]. The genomic region thought to contain HoxA11 and HoxA13 genes in each type of organism is represented under these panels. Note the regions encoding for alanine clusters, with a minimum of three residues (in red) and putative regulatory regions in blue. HoxA11AS are the long non-coding HoxA11 RNAs thought to have an inhibitory effect on HoxA11 transcription. Question marks in the basal gnathostome condition represent the lack of knowledge of putative regulatory regions operating on 5' HoxA genes. The basal actinopterygian condition was assembled based on data from spotted gar (Lepisosteus oculatus). Figure assembly based on $[1,40,41,43,57,66,67]$.

Thus, there is in fact grounds to speculate that the incorporation of polyalanine repeats in HoxA11 and HoxA13 proteins may have been an important step for the evolution of limbs. Although a remark has to be made regarding the alanine content of HoxA11 and HoxA13 proteins between fish, amphibians and birds as the comparative alanine content does not support a clear correlation between higher alanine percentage and the presence of an autopod (Figures 2 and 3). Two hypotheses may explain this observation. The first one is that the relatively similar alanine content of amphibians and birds in comparison with fish, but lower in comparison with mammals, results from the different evolutionary paths that those groups experienced [68]. Indeed, some reptiles and amphibians have a remarkable evolutionary history with reference to the evolution of autopod, with squamates having multiple autopod losses, estimated to have occurred at least 25 times independently [69]. Thus, for amphibians, reptiles and birds, a different molecular signature may be correlated with the evolution of the autopod [68]. The second hypothesis is that the contribution of alanines for autopod evolution is not merely quantitative, but also qualitative. However, functional assays are mandatory to make the proof of principle of these hypotheses, as the importance of alanine repeats for limb evolution remains largely unexplored. Taking advantage of the genome editing capacity of the CRISPR-Cas9 technology [70] and the lack of polyalanine repeats in zebrafish [40,41], it would be possible to introduce polyalanine repeats within the zebrafish HoxA11 or HoxA13 and assess how such sequences 
impact fin development and/or alter the way these transcription factors interact with their downstream targets. The realization of such experiments holds the potential of exploring how homopolymeric amino acid repeats may have impacted the fin-to-limb transition at quantitative and qualitative levels and, on a broader scale, provides evidence of how such repeat expansions may have contributed to the morphological plasticity in vertebrates.

\section{Non-Coding RNAs and the Fin-to-Limb Transition}

In the past decade, the scientific community has discovered that RNA's biological function is far more complex than the initially proposed role as simple mediators in the DNA-to-protein information flow [71]. Recently, several classes of non-coding RNAs (ncRNAs) with proven biological function have been discovered, such as: long-non-coding RNAs (lncRNAs), microRNAs (miRNAs), Piwi-interacting RNAs (piRNAs), small interfering RNAs (siRNAs), enhancer RNAs (eRNAs) and promoter-associated RNAs (PARs) [72]. Those ncRNAs have various functions, such as suppression of transposon activity and post-transcriptional gene silencing. They may also act as gene transcriptional activators or repressors and be involved in gene epigenetic control [72-74].

Although exceptions are known, overall, those RNAs seem to have two characteristics in common: a low transcriptional level and a high evolutionary divergence, with their numbers increasing in the genomes along with the species' evolutionary history [72,75]. For example, a recent study demonstrates that the majority of long-non-coding RNAs in each species are lineage specific [75]. This finding, along with the discovery that ncRNAs are important for proper gene regulation during development in some species [74,76], designates the ncRNAs as possible drivers of species morphological innovation [74].

Interestingly, the HOX loci produce a considerable repertoire of ncRNAs belonging to two major classes: miRNAs (e.g., mir-10, mir-196, mir-99, mir-126) and lncRNAs (e.g., HOTAIR, HOTTIP, Mistral, HOTATRM1) [77]. Those ncRNAs have been implicated in the regulation of HOX gene expression at the transcriptional, post-transcriptional and translational level [77], and a particularly interesting example of the contribution of such RNA species in the modulation of morphological design comes from mir-196, a well-conserved miRNA in vertebrates [76,78]. McGlinn and colleagues reported that knocking down mir-196 expression in chicken embryos caused a morphologic alteration of the last cervical vertebrae into a thoracic-like identity [76]. The phenotypic alteration seems to result partially from an expansion of the Hoxb8 expression domain, caused by the reduction of mir-196 transcripts [76]. This example shows how relevant miRNAs, and on a broader scale the ncRNAs, are for the regulation of important patterning genes during development [76], with possible evolutionary implications in the establishment of specific morphological traits.

In addition, these ncRNAs may also participate in the epigenetic control of transcription. The lncRNAs HOTAIR was the first one to be discovered to play that role, alerting the importance of RNAs as controllers of the transcriptional activity that may have been highly influential in the determination of the cell fate and, ultimately, in the evolution of animal morphology [79,80]. However, the versatility of functions that these lncRNAs perform during development and disease remains largely unknown. Distinct sources of information (such as conservation, coding potential patterns and anatomical properties) are currently being used to identify the main IncRNA gene families [80]. Regarding the anatomical properties, lncRNAs have been grouped, for example, as: (1) antisense IncRNAs that overlap protein-coding genes; (2) intronic lncRNAs that are encoded within introns of protein-coding genes; (3) divergent lncRNAs encoded in the opposite direction of a protein coding gene, but sharing its promoter; (4) intergenic lncRNAs that are encoded within the intergenic space between protein-coding loci (lincRNAs).

Among the Hox genes found to be involved in limb development, the HoxA11 locus stands out as capable of generating several antisense lncRNAs (HoxA11AS) in a variety of tetrapods $[42,57,74,81]$ that were found to be polyadenylated and alternately processed [42,57]. Moreover, comparison of these antisense HoxA11 transcripts revealed tissue-specific differences, as well as aspects of RNA processing 
conserved during evolution, which strongly suggest that the antisense RNAs from the Hoxa 11 locus are functionally significant [42].

Two of these HoxA11AS have overlapping complementary sequences with the first exon of HoxA11 (ENST00000520360.4 and ENST00000522863.1; Figure 4). In contrast, others seem to have sequence complementary with the enhancer/promoter region of HoxA11 and appear to lack sequence conservation between fish and mammals (e.g., ENST00000522674.1). In order to evaluate the antisense transcript expression of HoxA11AS, Hsieh-Li and colleagues performed in situ hybridizations using sense and antisense probes designed for the first exon of HoxA11 [57]. Surprisingly, they found complementary expression patterns of these probes in mouse embryonic limbs. At E9.5, the HoxA11 gene is transcribed throughout the distal limb bud region and then gets progressively proximally restricted, up to the point where the HoxA11 gene is only expressed in the prospective zeugopod region [42,57]. This progressive distal repression of the HoxA11 gene coincides with the appearance of the representatives of HoxA11AS transcripts that begin to be expressed in the most distal limb bud region at E10.5. This antisense expression domain then expands proximally, occupying the prospective autopod region by E11.5 [42,57]. The complementary expression patterns found between the sense and antisense probes designed against the HoxA11 first exon suggest that production of HoxA11AS transcripts during limb development may conduct expression inhibition of HoxA11 [42,57].

Cross species alignments using the "Comparative Genomic" tool from Ensemble (release 80, May 2015, [82]) suggest that the putative HoxA11AS encoding regions have been subjected to a different selective pressure during vertebrates' evolution. Indeed, the regions that overlap with the complementary sequence of $\operatorname{Hox} A 11$, in the first exon, seem to be significantly conserved among tetrapods. However, another HoxA11AS encoding region, which seems to be complementary to the HoxA11 enhancer/promoter region (ENST00000522674.1), is apparently less conserved [74]. Within mammals, this particular HoxA11 antisense is detectable in eutherians and is expressed during embryonic development, but also in the endometrium during pregnancy [74]. The potential role of this HoxA11AS in mammal reproduction may justify its generalized presence within eutherians' genomes. In non-mammal organisms, such as in the frog, the degree of sequence conservation drops to less than $50 \%$ [74]. Thus, this amphibian lncRNA, if functional, might be exclusively associated with the regulation of limb development and, as such, under lower selective pressure. Moreover, cross species alignment also revealed an absence of significant similarity between human HOXA11AS gene and the syntenic zebrafish, stickleback and coelacanth regions. Moreover, when Bartel and colleagues characterized the lncRNAs during zebrafish development using chromatin marks, poly(A)-site mapping and RNA-Seq data, they did not find any potential homologues of HOXA11AS, either by sequence conservation or genomic location [83]. This might be due to the striking lack of conservation of the teleost HoxA intergenic regions, when compared to the ones from other gnathostome lineages [84]. In fact, teleosts have duplicated and more compact HoxA clusters, with shorter intergenic regions [84], which may suggest that they lack most of the genomic region able to transcribe HoxA11AS. It remains to be uncovered if fish species that diverge prior to teleost radiation and where the length of the HoxA intergenic regions more closely resemble the ones from the tetrapods have the capacity to produce these lncRNAs (e.g., shark, paddlefish).

Given the possible inhibitory effect of HoxA11AS on HoxA11 and what seems to be a tetrapod-specific characteristic of HoxA11AS, we present the hypothesis that the evolutionary origin of HoxA11AS was one of the molecular events that might have contributed to the evolution of the fin/limb morphology. According to this hypothesis, the appearance of HoxA11AS expression in the distal limb region inhibits HoxA11 expression in that limb domain, thus uncoupling HoxA11 and HoxA13 limb expression domains and enabling the definition of a distal HoxA13 single positive autopod domain. The apparent lack of HoxA11AS homologues in the zebrafish, according to our hypothesis, may help to explain why the expression domains of HoxA11/HoxA13 do not split during fin development. In contrast, the transient separation of these domains detected during shark and paddlefish fin development $[26,35,36]$ suggests that these organisms may produce HoxA11AS. However, 
these HoxA11AS are probably transcribed in the antisense direction within their own HoxA11 loci; therefore, they may not be conserved among vertebrates. Taken as inspiration the work from Hsieh-Li and colleagues [57], it would be interesting to analyze the expression pattern of sense and antisense HoxA11 transcripts in these organisms.

An important step to gain insight into our preferential hypothesis, which relates the origin of HoxA11AS with the evolution of vertebrate fins, is to functionally characterize these transcripts to better understand their biological relevance to limb development. To achieve this goal, a possible strategy would be to take advantage of the chick model, which seems to have conserved HoxA11AS, and induce its overexpression, knockdown and knockout in ovo, following the strategy used by McGlinn and colleagues [76]. This would allow testing the impact of HoxA11AS in the definition of the HoxA11 expression domain during the formation of limbs. Another important step to explore our hypothesis is to assess the evolutionary importance of these lncRNAs during vertebrate fin/limb development. To this end, an innovative strategy would be the knock-in of HoxA11AS in zebrafish and the characterization of its impact during fin development, assuming that the capacity to metabolize these lncRNAs was assembled prior to the divergence of tetrapods.

\section{Cis-Regulation of 5' HoxA Genes and the Fin-to-Limb Transition}

According to the previously-presented hypothesis, the definition of the autopod domain is associated with the proximal restriction of HoxA11 expression during the development of appendicular structures in stem groups of tetrapods [26]. However, the autopod morphology, regarding digit size and number, acquired modifications during evolution probably due to an increase in the transcription of 5' HoxA and HoxD genes $[8,85,86]$. Thus, recruitment of novel non-coding regulatory elements, which are able to modulate the transcription of these genes, seems to have been a crucial step for the evolution of the autopod [85,86]. The enhancer elements that govern the expression of 5' HoxD have been characterized during the development of mouse limbs $[85,86]$. These reports highlighted that a particular combination of regulatory elements is required for the specification of the appropriate time, dosage and pattern of $5^{\prime}$ HoxD gene expression.

Regarding the HoxA11 and HoxA13 genes, despite their crucial role for the correct development and proximo-distal patterning of the limbs, information on their cis-regulatory network is still scarce, although the research teams led by Innis [47], Kmita [66], Gómez-Skarmeta and Shubin [43] have shed some light into the enhancers that drive HoxA13 expression in the developing limbs and their possible impact in the fin-to-limb transition. From the works published to date on mice, the enhancer network that regulates distal HoxA13 expression in the developing limbs is comprised of at least nineteen different elements located upstream of, and excluding, the HoxA cluster (Figure 4) [66]. Some of those enhancers are present within genes located upstream of the HoxA cluster, namely the Hibadh, Tax1bp1 and Jazf1 genes [66]. A curious observation is that the enhancers driving HoxA13 distal expression in mouse limb form several sub-megabase topological domains (sub-TADs) that contact each other in a fashion that, in some cases, seems to be independent of the enhancer's transcriptional activity. This suggests that the sub-TADs organization is governed by a developmental structure/function interplay instead of a mere clustering of active enhancers [66].

When the conservation of the mouse HoxA13 enhancer network was assayed, using sequence homology and ATAC-Seq (assay for transposase-accessible chromatin using sequencing) in zebrafish and gar, only the gar genome showed conservation of three (e10, e13 and e16) of the 19 enhancers that regulate HoxA13 transcription in mouse limb, and at least the gar e16 region induces, in zebrafish and mouse transgene reporter assays, an expression pattern that is very similar to the one driven by mouse e16 [43]. Taking into account that lack of sequence conservation is not necessarily sufficient to exclude the presence of enhancers in fish, these data nevertheless suggest two possible circumstances. Firstly, the zebrafish hoxa13a and hoxa13b enhancer network, if present, represents a derived state, as no sequence homology was detected between zebrafish, gar and mouse HoxA enhancers [43]. This observation may result from extensive remodeling of HoxA regulatory elements caused by the 
occurrence of a third whole genome duplication in teleosts followed by gene sub-functionalization, thus leading to the inability of the zebrafish hoxa13 paralogs to respond to a "distal limb program" during development [43]. Secondly, the enhancer network that regulates HoxA13's late phase expression suffered extensive elaboration/remodeling over the course of the vertebrates' evolution, although some of its elements might have already been present in the last common ancestor of bony vertebrates [43]. The extensive elaboration of HoxA13 enhancer elements throughout vertebrate evolution suggests that the fish HoxA13 transcriptional level during fin development is too low to elicit an adequate "distal limb identity", thus suggesting that HoxA13 gene dosage increase was a requirement for the fin-to-limb transition. This hypothesis finds support in the work published, in 1997, by Zákány and colleagues [8], where by analyzing a set of distal HoxA and HoxD compound mutants, this team found that overall autopod morphology, and in particular digit number and length, greatly varies in response to Hox dosage [8] and to a minor extent to the qualitative nature of the expressed HoxA and HoxD proteins (Hox code) [85]. Moreover, overexpression of zebrafish hoxd13a was shown to cause changes in the morphogenesis of the fins towards a limb-like identity [22]. Testing out the hypothesis that an increase of HoxA13 dosage was an evolutionary requirement for the fin-to-limb transition could be easily achieved in zebrafish following a strategy previously described by Freitas and colleagues [22]. Performing such a proof-of-principle study not only upholds the potential to disclose how increasing levels of HoxA13 proteins may have contributed to the morphological transition between fish fins and tetrapod limbs, but may also shed light on how regulation and function of Hox13 paralogs genes may have set the grounds for autopod evolution.

\section{Conclusions and Future Directions}

In this review, we attempted to survey the evolution of HoxA11 and HoxA13 transcription factors and their regulatory control in vertebrates. Evaluating the current state of the art and performing complementary analyses, using information currently available for distinct vertebrate lineages, we support the hypotheses implicating changes in the alanine content, capacity to generate long-non-coding RNAs and incorporation of a novel cis-regulatory region in the developmental mechanisms involved in the fin-to-limb transition in vertebrates (Figure 4).

Conserved polyalanine tracts with a minimum of four residues are common within the HoxA11 and HoxA13 proteins of tetrapods (Figures 2-4). However, these types of sequences are undetectable in the orthologs of fish. In the HoxA11 proteins, these enriched alanine domains co-localize with the hydrophilic region, being higher the percentages of alanines detectable in amniotes. Among the fish analyzed, the hydrophilic region of paddlefish seems to be the one that has a higher content of alanines. These observations point us to two hypotheses: (1) an increased alanine content within HoxA11 proteins may have impacted the formation of specific amniote limb structures during evolution, such as the hand plates that precede digit differentiation; (2) incorporation of extra alanine residues into the hydrophilic regions of HoxA11 protein probably started prior to the tetrapod divergence, in the common ancestor of actinopterygians and sarcopterygians. In the HoxA13 proteins, most polyalanine tracts (I-VII) are conserved in mammals (Figures 3 and 4). However, the polyalanine Tract III seems to be present in the chick HoxA13 proteins, which may suggest a particular role of these tracts in the formation of amniote-specific limb structures, the hand plates.

Taking into consideration the functional potential found for this type of amino acid repeats $[40,47,49]$, their integration into the HoxA11 and Hoxa13 proteins during evolution might have modified the way they interact with their downstream targets, contributing to the phenotypic variation found in the evolutionary transition from fish fins to tetrapod limbs. For example, the polyalanine repeats found in the HoxA11 and HoxA13 proteins might have enhanced their repressor role upon other molecules, helping to establish novel domains of cell identity, leading to the differentiation of the hand plate in amniotes. The repressor potential of HoxA13 in particular might have inhibited the expression of HoxA11 in the most distal mesenchyme of the appendages, generating a novel field of cell identity, which might have potentiated the formation of additional skeletal elements. However, 
the proof of principle of these hypotheses is lacking. Here, we suggest, as a future working perspective, the use of CRISPR-Cas9 technology [70] to harbor polyalanine repeats within the zebrafish HoxA11 or HoxA13 in order to evaluate the phenotypic consequences of such sequences during appendage development. These experiments would certainly add to our knowledge of how changes in the alanine content of transcription factors impacted the diversification of vertebrate structures.

In addition, we show here that the capacity to generate HoxA11AS, which are long-non-coding RNAs, seems to be exclusive to tetrapods (Figure 4) [42,57,74,81]. Moreover the expression of particular HoxA11 antisense RNAs was found to be complementary to HoxA11, suggesting that while HoxA11 is progressively repressed distally, HoxA11AS expression expands proximally. This observation points to a repressor role of HoxA11AS on HoxA11 that might have contributed to split the HoxA11/HoxA13 expression domains, potentiating the formation of the zeugopod and the autopod. Given that during catshark and paddlefish fin development, the expression domains of hoxa11 and hoxa13 also split transiently into two non-overlapping territories, it would be important to search for HoxA11AS homologues in these organisms and evaluate the potential repressor role on HoxA11. These ideas also lack functional validation, and a first step would be to gain more insight into the expression of HoxA11 antisense in fish representing distinct phylogenetic positions. Moreover, it would be important to better characterize the role of HoxA11AS during tetrapod limb development, to test their developmental and evolutionary potential harboring of HoxA11AS in zebrafish and to characterize the impact during appendage development.

Finally, we suggest that the transcriptional levels of HoxA11 and HoxA13 might have also influenced appendage evolution, as has been proposed for HoxD13 [22]. Indeed, the autopod morphology in mouse, in particular digit number and length, is influenced by the Hox dosage [8], which often depends on the enhancer capacity of cis-regulatory regions within their genomic landscape [87]. Marked differences in the putative regulatory regions of the $5^{\prime}$ HoxA genes were identified in fish species representing distinct phylogenetic positions, such as the spotted gar and the zebrafish (Figure 4). Thus, cis-regulatory changes may have interfered with the differential fin morphology of distinct fish groups (Figure 4). Within tetrapods, subtle changes were also identified in the putative regulatory regions of these genes that may have contributed to the distinct autopod anatomy found in mammals and birds, for example [43]. Additionally, fish versus tetrapod comparisons suggest a significant increase of cis-regulatory modules in tetrapods [43], a phenomena that might have been crucial to achieve the appropriate levels of Hox expression required for the origin of the autopod. To further explore the consequences of increased levels of $5^{\prime}$ HoxA proteins and the addition of novel regulatory modules for the developmental processes resulting in the evolution of limbs, we emphasize the advantages of the zebrafish model to perform the appropriate proof of principle studies due to its current technical repertoire, allowing a variety of functional assays [22].

Acknowledgments: We are grateful to Mafalda Araújo for aid in phylogenetic analyses. We also acknowledge the financial support of "Fundação para a Ciência e Tecnologia" (FCT-Portugal) through the research project EXPL/BEX-BID/0801/2013.

Author Contributions: J.L.C. conceived of and wrote the manuscript. V.B. conceived of the artwork and collaborated in figure assembly. P.N.R. contributed to the discussion. R.F. participated in the writing, figure assembly and overall supervision of the work. All authors commented on the manuscript at distinct stages.

Conflicts of Interest: The authors declare no conflict of interest.

\section{References}

1. Shubin, N.; Tabin, C.; Carroll, S. Deep homology and the origins of evolutionary novelty. Nature 2009, 457, 818-823. [CrossRef] [PubMed]

2. Coates, M.I.; Jeffery, J.E.; Rut, M. Fins to limbs: What the fossils say. Evol. Dev. 2002, 4, 390-401. [CrossRef] [PubMed]

3. Shubin, N. The evolution of paired fins and the origin of tetrapod limbs-Phylogenetic and transformational approaches. Evol. Biol. 1995, 28, 39-86. 
4. Janvier, P. Early Vertebrates; Oxford University Press: Oxford, UK, 1996.

5. Boisvert, C.A.; Mark-Kurik, E.; Ahlberg, P.E. The pectoral fin of Panderichthys and the origin of digits. Nature 2008, 456, 636-638. [CrossRef] [PubMed]

6. Coates, M.I.; Clack, J.A. Polydactyly in the earliest known tetrapod limbs. Nature 1990, 347, 66-68. [CrossRef]

7. Coates, M.I. The origin of vertebrate limbs. Dev. Suppl. 1994, 169-180.

8. Zakany, J.; Fromental-Ramain, C.; Warot, X.; Duboule, D. Regulation of number and size of digits by posterior Hox genes: A dose-dependent mechanism with potential evolutionary implications. Proc. Natl. Acad. Sci. USA 1997, 94, 13695-13700. [CrossRef] [PubMed]

9. Delgado, I.; Torres, M. Gradients, waves and timers, an overview of limb patterning models. Semin. Cell Dev. Biol. 2016. [CrossRef] [PubMed]

10. Zeller, R.; Lopez-Rios, J.; Zuniga, A. Vertebrate limb bud development: Moving towards integrative analysis of organogenesis. Nat. Rev. Genet. 2009, 10, 845-858. [CrossRef] [PubMed]

11. Mariani, F.V.; Ahn, C.P.; Martin, G.R. Genetic evidence that FGFs have an instructive role in limb proximal-distal patterning. Nature 2008, 453, 401-456. [CrossRef] [PubMed]

12. Cooper, K.L.; Hu, J.K.; ten Berge, D.; Fernandez-Teran, M.; Ros, M.A.; Tabin, C.J. Initiation of proximal-distal patterning in the vertebrate limb by signals and growth. Science 2011, 332, 1083-1086. [CrossRef] [PubMed]

13. Grandel, H.; Schulte-Merker, S. The development of the paired fins in the zebrafish (Danio rerio). Mech. Dev. 1998, 79, 99-120. [CrossRef]

14. Nakamura, T.; Klomp, J.; Pieretti, J.; Schneider, I.; Gehrke, A.R.; Shubin, N.H. Molecular mechanisms underlying the exceptional adaptations of batoid fins. Proc. Natl. Acad. Sci. USA 2015, 112, 15940-15945. [CrossRef] [PubMed]

15. Yano, T.; Abe, G.; Yokoyama, H.; Kawakami, K.; Tamura, K. Mechanism of pectoral fin outgrowth in zebrafish development. Development 2012, 139, 2916-2925. [CrossRef] [PubMed]

16. Ohgo, S.; Itoh, A.; Suzuki, M.; Satoh, A.; Yokoyama, H.; Tamura, K. Analysis of hoxa11 and hoxa13 expression during patternless limb regeneration in Xenopus. Dev. Biol. 2010, 338, 148-157. [CrossRef] [PubMed]

17. Ahn, D.; Ho, R.K. Tri-phasic expression of posterior Hox genes during development of pectoral fins in zebrafish: Implications for the evolution of vertebrate paired appendages. Dev. Biol. 2008, 322, 220-233. [CrossRef] [PubMed]

18. Sheth, R.; Marcon, L.; Bastida, M.F.; Junco, M.; Quintana, L.; Dahn, R.; Kmita, M.; Sharpe, J.; Ros, M.A. Hox genes regulate digit patterning by controlling the wavelength of a turing-type mechanism. Science 2012, 338, 1476-1480. [CrossRef]

19. Tarchini, B.; Duboule, D.; Kmita, M. Regulatory constraints in the evolution of the tetrapod limb anterior-posterior polarity. Nature 2006, 443, 985-988. [CrossRef] [PubMed]

20. Dolle, P.; Dierich, A.; Lemeur, M.; Schimmang, T.; Schuhbaur, B.; Chambon, P.; Duboule, D. Disruption of the Hoxd-13 gene induces localized heterochrony leading to mice with neotenic limbs. Cell 1993, 75, 431-441. [CrossRef]

21. Sordino, P.; van der Hoeven, F.; Duboule, D. Hox gene expression in teleost fins and the origin of vertebrate digits. Nature 1995, 375, 678-681. [CrossRef] [PubMed]

22. Freitas, R.; Gomez-Marin, C.; Wilson, J.M.; Casares, F.; Gomez-Skarmeta, J.L. Hoxd13 contribution to the evolution of vertebrate appendages. Dev. Cell. 2012, 23, 1219-1229. [CrossRef] [PubMed]

23. Nelson, C.E.; Morgan, B.A.; Burke, A.C.; Laufer, E.; DiMambro, E.; Murtaugh, L.C.; Gonzales, E.; Tessarollo, L.; Parada, L.F.; Tabin, C. Analysis of Hox gene expression in the chick limb bud. Development 1996, 122, 1449-1466. [PubMed]

24. Perez, W.D.; Weller, C.R.; Shou, S.M.; Stadler, H.S. Survival of Hoxa13 homozygous mutants reveals a novel role in digit patterning and appendicular skeletal development. Dev. Dyn. 2010, 239, 446-457. [CrossRef] [PubMed]

25. Knosp, W.M.; Scott, V.; Bachinger, H.P.; Stadler, H.S. HOXA13 regulates the expression of bone morphogenetic proteins 2 and 7 to control distal limb morphogenesis. Development 2004, 131, 4581-4592. [CrossRef] [PubMed]

26. Metscher, B.D.; Takahashi, K.; Crow, K.; Amemiya, C.; Nonaka, D.F.; Wagner, G.P. Expression of Hoxa-11 and Hoxa-13 in the pectoral fin of a basal ray-finned fish, Polyodon spathula: Implications for the origin of tetrapod limbs. Evol. Dev. 2005, 7, 186-195. [CrossRef] [PubMed] 
27. Yokouchi, Y.; Nakazato, S.; Yamamoto, M.; Goto, Y.; Kameda, T.; Iba, H.; Kuroiwa, A. Misexpression of Hoxa-13 induces cartilage homeotic transformation and changes cell adhesiveness in chick limb buds. Genes Dev. 1995, 9, 2509-2522. [CrossRef] [PubMed]

28. Yokouchi, Y.; Ohsugi, K.; Sasaki, H.; Kuroiwa, A. Chicken Homeobox gene Msx-1-Structure, expression in limb buds and effect of retinoic acid. Development 1991, 113, 431-444. [PubMed]

29. Yokouchi, Y.; Sasaki, H.; Kuroiwa, A. Homeobox gene-expression correlated with the bifurcation process of limb cartilage development. Nature 1991, 353, 443-445. [CrossRef]

30. Mercader, N.; Leonardo, E.; Azpiazu, N.; Serrano, A.; Morata, G.; Martínez, C.; Torres, M. Conserved regulation of proximodistal limb axis development by Meis1/Hth. Nature 1999, 402, 425-429. [PubMed]

31. Mercader, N.; Selleri, L.; Criado, L.M.; Pallares, P.; Parras, C.; Cleary, M.L.; Torres, M. Ectopic Meis1 expression in the mouse limb bud alters P-D patterning in a Pbx1-independent manner. Int. J. Dev. Biol. 2009, 53, 1483-1494. [CrossRef] [PubMed]

32. Sato, K.; Koizumi, Y.; Takahashi, M.; Kuroiwa, A.; Tamura, K. Specification of cell fate along the proximal-distal axis in the developing chick limb bud. Development 2007, 134, 1397-1406. [CrossRef] [PubMed]

33. Tabin, C.; Wolpert, L. Rethinking the proximodistal axis of the vertebrate limb in the molecular era. Genes Dev. 2007, 21, 1433-1442. [CrossRef] [PubMed]

34. Neumann, C.J.; Grandel, H.; Gaffield, W.; Schulte-Merker, S.; Nusslein-Volhard, C. Transient establishment of anteroposterior polarity in the zebrafish pectoral fin bud in the absence of sonic hedgehog activity. Development 1999, 126, 4817-4826. [PubMed]

35. Sakamoto, K.; Onimaru, K.; Munakata, K.; Suda, N.; Tamura, M.; Ochi, H.; Tanaka, M. Heterochronic shift in Hox-mediated activation of sonic hedgehog leads to morphological changes during fin development. PLoS ONE 2009, 4, e5121. [CrossRef] [PubMed]

36. Davis, M.C.; Dahn, R.D.; Shubin, N.H. An autopodial-like pattern of Hox expression in the fins of a basal actinopterygian fish. Nature 2007, 447, 473-476. [CrossRef] [PubMed]

37. Yano, T.; Tamura, K. The making of differences between fins and limbs. J. Anat. 2013, 222, 100-113. [CrossRef]

38. Freitas, R.; Gomez-Skarmeta, J.L.; Rodrigues, P.N. New frontiers in the evolution of fin development. J. Exp. Zool. B Mol. Dev. Evol. 2014, 322, 540-552. [CrossRef] [PubMed]

39. Roensch, K.; Tazaki, A.; Chara, O.; Tanaka, E.M. Progressive specification rather than intercalation of segments during limb regeneration. Science 2013, 342, 1375-1379. [CrossRef] [PubMed]

40. Mortlock, D.P.; Sateesh, P.; Innis, J.W. Evolution of N-terminal sequences of the vertebrate HOXA13 protein. Mamm. Genome 2000, 11, 151-158. [CrossRef]

41. Chiu, C.H.; Nonaka, D.; Xue, L.; Amemiya, C.T.; Wagner, G.P. Evolution of Hoxa-11 in lineages phylogenetically positioned along the fin-limb transition. Mol. Phylogenet. Evol. 2000, 17, 305-316. [CrossRef] [PubMed]

42. Potter, S.S.; Branford, W.W. Evolutionary conservation and tissue-specific processing of Hoxa 11 antisense transcripts. Mamm. Genome 1998, 9, 799-806. [CrossRef] [PubMed]

43. Gehrke, A.R.; Schneider, I.; de la Calle-Mustienes, E.; Tena, J.J.; Gomez-Marin, C.; Chandran, M.; Nakamura, T.; Braasch, I.; Postlethwait, J.H.; Gómez-Skarmeta, J.L.; et al. Deep conservation of wrist and digit enhancers in fish. Proc. Natl. Acad. Sci. USA 2015, 112, 803-808. [CrossRef]

44. Rorick, M.M.; Wagner, G.P. The origin of conserved protein domains and amino acid repeats via adaptive competition for control over amino acid residues. J. Mol. Evol. 2010, 70, 29-43. [CrossRef] [PubMed]

45. Mularoni, L.; Ledda, A.; Toll-Riera, M.; Alba, M.M. Natural selection drives the accumulation of amino acid tandem repeats in human proteins. Genome Res. 2010, 20, 745-754. [CrossRef] [PubMed]

46. Fondon, J.W.; Garner, H.R. Molecular origins of rapid and continuous morphological evolution. Proc. Natl. Acad. Sci. USA 2004, 101, 18058-18063. [CrossRef] [PubMed]

47. Lehoczky, J.A.; Innis, J.W. Expanded HOXA13 polyalanine tracts in a monotreme. Evol. Dev. 2008, 10, 433-438. [CrossRef] [PubMed]

48. HannaRose, W.; Hansen, U. Active repression mechanisms of eukaryotic transcription repressors. Trends Genet. 1996, 12, 229-234. [CrossRef]

49. Lavoie, H.; Debeane, F.; Trinh, Q.D.; Turcotte, J.F.; Corbeil-Girard, L.P.; Dicaire, M.J.; Saint-Denis, A.; Pagé, M.; Rouleau, G.A.; Brais, B. Polymorphism, shared functions and convergent evolution of genes with sequences coding for polyalanine domains. Hum. Mol. Genet. 2003, 12, 2967-2979. [CrossRef] [PubMed] 
50. Frisen, L.; Lagerstedt, K.; Tapper-Persson, M.; Kockum, I.; Nordenskjold, A. A novel duplication in the HOXA13 gene in a family with atypical hand-foot-genital syndrome. J. Med. Genet. 2003, 40, e49. [CrossRef]

51. Anan, K.; Yoshida, N.; Kataoka, Y.; Sato, M.; Ichise, H.; Nasu, M.; Ueda, S. Morphological change caused by loss of the taxon-specific polyalanine tract in Hoxd-13. Mol. Biol. Evol. 2007, 24, 281-287. [CrossRef] [PubMed]

52. Johnson, K.R.; Sweet, H.O.; Donahue, L.R.; Ward-Bailey, P.; Bronson, R.T.; Davisson, M.T. A new spontaneous mouse mutation of Hoxd13 with a polyalanine expansion and phenotype similar to human synpolydactyly. Hum. Mol. Genet. 1998, 7, 1033-1038. [CrossRef] [PubMed]

53. Bruneau, S.; Johnson, K.R.; Yamamoto, M.; Kuroiwa, A.; Duboule, D. The mouse Hoxd13(spdh) mutation, a polyalanine expansion similar to human type II synpolydactyly (SPD), disrupts the function but not the expression of other Hoxd genes. Dev. Biol. 2001, 237, 345-353. [CrossRef] [PubMed]

54. Goodman, F.R. Limb malformations and the human HOX genes. Am. J. Med. Genet. 2002, 112, $256-265$. [CrossRef]

55. Mortlock, D.P.; Innis, J.W. Mutation of HOXA13 in hand-foot-genital syndrome. Nat. Genet. 1997, 15, $179-180$. [CrossRef] [PubMed]

56. Innis, J.W.; Mortlock, D.; Chen, Z.; Ludwig, M.; Williams, M.E.; Williams, T.M.; Doyle, C.D.; Shao, Z.; Glynn, M.; Mikulic, D.; et al. Polyalanine expansion in HOXA13: Three new affected families and the molecular consequences in a mouse model. Hum. Mol. Genet. 2004, 13, 2841-2851. [CrossRef] [PubMed]

57. Hsieh-Li, H.M.; Witte, D.P.; Weinstein, M.; Branford, W.; Li, H.; Small, K.; Potter, S.S. Hoxa 11 structure, extensive antisense transcription, and function in male and female fertility. Development 1995, 121, 1373-1385. [PubMed]

58. Larkin, M.A.; Blackshields, G.; Brown, N.P.; Chenna, R.; McGettigan, P.A.; McWilliam, H.; Valentin, F.; Wallace, I.M.; Wilm, A.; Lopez, R.; et al. Clustal W and Clustal X version 2.0. Bioinformatics 2007, 23, 2947-2948. [CrossRef] [PubMed]

59. Torok, M.A.; Gardiner, D.M.; Shubin, N.H.; Bryant, S.V. Expression of HoxD genes in developing and regenerating axolotl limbs. Dev. Biol. 1998, 200, 225-233. [CrossRef]

60. Keenan, S.R.; Beck, C.W. Xenopus limb bud morphogenesis. Dev. Dyn. 2016, 245, 233-243. [CrossRef] [PubMed]

61. Handrigan, G.R.; Wassersug, R.J. The anuran Bauplan: A review of the adaptive, developmental, and genetic underpinnings of frog and tadpole morphology. Biol. Rev. Camb. Philos. Soc. 2007, 82, 1-25. [CrossRef] [PubMed]

62. Mitchell, P.J.; Tjian, R. Transcriptional regulation in mammalian-cells by sequence-specific dna-binding proteins. Science 1989, 245, 371-378. [CrossRef]

63. Roth, J.J.; Breitenbach, M.; Wagner, G.P. Repressor domain and nuclear localization signal of the murine Hoxa-11 protein are located in the homeodomain, no evidence for role of poly alanine stretches in transcriptional repression. J. Exp. Zool. B Mol. Dev. Evol. 2005, 304, 468-475. [CrossRef] [PubMed]

64. Mock, B.A.; D'Hoostelaere, L.A.; Matthai, R.; Huppi, K. A mouse homeo box gene, Hox-1.5, and the morphological locus, Hd, map to within $1 \mathrm{cM}$ on chromosome 6. Genetics 1987, 116, 607-612. [PubMed]

65. Schnabel, C.A.; AbateShen, C. Repression by HoxA7 is mediated by the homeodomain and the modulatory action of its N-terminal-arm residues. Mol. Cell. Biol. 1996, 16, 2678-2688. [CrossRef] [PubMed]

66. Berlivet, S.; Paquette, D.; Dumouchel, A.; Langlais, D.; Dostie, J.; Kmita, M. Clustering of tissue-specific sub-TADs accompanies the regulation of HoxA genes in developing limbs. PLoS Genet. 2013, 9, e1004018. [CrossRef] [PubMed]

67. Schneider, I.; Shubin, N.H. The origin of the tetrapod limb: From expeditions to enhancers. Trends Genet. 2013, 29, 419-426. [CrossRef] [PubMed]

68. Kohlsdorf, T.; Cummings, M.P.; Lynch, V.J.; Stopper, G.F.; Takahashi, K.; Wagner, G.P. A molecular footprint of limb loss: Sequence variation of the autopodial identity gene Hoxa-13. J. Mol. Evol. 2008, 67, 581-593. [CrossRef] [PubMed]

69. Wiens, J.J.; Brandley, M.C.; Reeder, T.W. Why does a trait evolve multiple times within a clade? Repeated evolution of snakelike body form in squamate reptiles. Evolution 2006, 60, 123-141. [CrossRef] [PubMed]

70. Jao, L.E.; Wente, S.R.; Chen, W. Efficient multiplex biallelic zebrafish genome editing using a CRISPR nuclease system. Proc. Natl. Acad. Sci. USA 2013, 110, 13904-13909. [CrossRef] [PubMed] 
71. Ponting, C.P.; Oliver, P.L.; Reik, W. Evolution and functions of long noncoding RNAs. Cell 2009, 136, 629-641. [CrossRef] [PubMed]

72. Kaikkonen, M.U.; Lam, M.T.Y.; Glass, C.K. Non-coding RNAs as regulators of gene expression and epigenetics. Cardiovasc. Res. 2011, 90, 430-440. [CrossRef] [PubMed]

73. Mattick, J.S. Non-coding RNAs: The architects of eukaryotic complexity. EMBO Rep. 2001, 2, $986-991$. [CrossRef] [PubMed]

74. Yu, H.S.; Lindsay, J.; Feng, Z.P.; Frankenberg, S.; Hu, Y.; Carone, D.; Shaw, G.; Pask, A.J.; O'Neill, R.; Papenfuss, A.T.; et al. Evolution of coding and non-coding genes in HOX clusters of a marsupial. BMC Genomics 2012, 13. [CrossRef] [PubMed]

75. Hezroni, H.; Koppstein, D.; Schwartz, M.G.; Avrutin, A.; Bartel, D.P.; Ulitsky, I. Principles of long noncoding RNA evolution derived from direct comparison of transcriptomes in 17 species. Cell Rep. 2015, 11, 1110-1122. [CrossRef]

76. McGlinn, E.; Yekta, S.; Mansfield, J.H.; Soutschek, J.; Bartel, D.P.; Tabin, C.J. In ovo application of antagomiRs indicates a role for miR-196 in patterning the chick axial skeleton through Hox gene regulation. Proc. Natl. Acad. Sci. USA 2009, 106, 18610-18615. [CrossRef] [PubMed]

77. Leite-Castro, J.; Rodrigues, P.N.; Freitas, R. Hox gene regulation in vertebrates. Trends Dev. Biol. 2014, 8, 77-95.

78. Olena, A.F.; Patton, J.G. Genomic organization of microRNAs. J. Cell. Physiol. 2010, 222, 540-545. [CrossRef] [PubMed]

79. Rinn, J.L.; Kertesz, M.; Wang, J.K.; Squazzo, S.L.; Xu, X.; Brugmann, S.A.; Goodnough, L.H.; Helms, J.A.; Farnham, P.J.; Segal, E.; et al. Functional demarcation of active and silent chromatin domains in human HOX loci by noncoding RNAs. Cell 2007, 129, 1311-1323. [CrossRef] [PubMed]

80. Rinn, J.L.; Chang, H.Y. Genome regulation by long noncoding RNAs. Annu. Rev. Biochem. 2012, 81, $145-166$. [CrossRef] [PubMed]

81. Bodenmiller, D.M.; Baxter, C.S.; Hansen, D.V.; Potter, S.S. Phylogenetic analysis of Hoxa 11 sequences reveals absence of transposable elements, conservation of transcription factor binding sites, and suggests antisense coding function. DNA Sequence 2002, 13, 77-83. [CrossRef] [PubMed]

82. Flicek, P.; Amode, M.R.; Barrell, D.; Beal, K.; Billis, K.; Brent, S.; Carvalho-Silva, D.; Clapham, P.; Coates, G.; Fitzgerald, S.; et al. Ensembl 2014. Nucleic Acids Res. 2014, 42, D749-D755.

83. Ulitsky, I.; Shkumatava, A.; Jan, C.H.; Sive, H.; Bartel, D.P. Conserved function of lincRNAs in vertebrate embryonic development despite rapid sequence evolution. Cell 2011, 147, 1537-1550. [CrossRef] [PubMed]

84. Prohaska, S.J.; Fried, C.; Flamm, C.; Wagner, G.P.; Stadler, P.F. Surveying phylogenetic footprints in large gene clusters: Applications to Hox cluster duplications. Mol. Phylogenet. Evol. 2004, 31, 581-604. [CrossRef] [PubMed]

85. Montavon, T.; le Garrec, J.F.; Kerszberg, M.; Duboule, D. Modeling Hox gene regulation in digits: Reverse collinearity and the molecular origin of thumbness. Genes Dev. 2008, 22, 346-359. [CrossRef] [PubMed]

86. Woltering, J.M.; Noordermeer, D.; Leleu, M.; Duboule, D. Conservation and divergence of regulatory strategies at Hox Loci and the origin of tetrapod digits. PLoS Biol. 2014, 12, e1001773. [CrossRef] [PubMed]

87. Andrey, G.; Duboule, D. SnapShot: Hox gene regulation. Cell 2014, 156. [CrossRef] [PubMed]

(C) 2016 by the authors; licensee MDPI, Basel, Switzerland. This article is an open access article distributed under the terms and conditions of the Creative Commons by Attribution (CC-BY) license (http:/ / creativecommons.org/licenses/by/4.0/). 